\title{
Application of Intensive Pain Nursing in Postoperative Nursing of Clinical Patients with Lung Cancer
}

\author{
Fangming Sun* \\ Zhenjiang First People's Hospital, Zhenjiang 212002, Jiangsu Province, China \\ *Corresponding author: Fangming Sun, 115526194@qq.com
}

\begin{abstract}
Objective: The intensive pain nursing was added to the surgical nursing of lung cancer and the clinical nursing effect was discussed. Methods: 50 patients with lung cancer received in our hospital from January 2020 to June 2021. The observation group was given intensive pain nursing and the control group was given routine nursing. The clinical nursing effects of the two groups were studied. Results: The pain degree of the two groups was improved after nursing. The pain relief effect of the observation group was higher than that of the control group, and the nursing effect of the two groups was different $(P<0.05)$. Conclusion: In the postoperative nursing of patients with lung cancer, the intervention of intensive pain nursing can relieve the pain of patients, which is worth popularizing.
\end{abstract}

Keywords: Intensive pain nursing; Lung cancer surgery; Application

Publication date: September 2021; Online publication: September 30, 2021

\section{Introduction}

Lung cancer is the fastest-growing malignant disease in China in recent 30 years. It is also a common primary malignant tumor in clinic, which seriously threatens the health and life safety of our people. At present, the development of medical science and technology has not developed specific drugs for lung cancer, the most effective clinical treatment for lung cancer is surgery. The main complaint of lung cancer patients after surgery is pain, which requires nursing intervention. If the patient's pain nursing is not ideal, it may cause the patient's slow postoperative recovery and affect the patient's entry into normal life. This paper mainly takes intensive pain nursing to intervene the postoperative recovery stage of patients, and studies the application effect of this nursing.

\section{Materials and methods}

\subsection{General information}

50 patients with lung cancer received in our hospital from January 2020 to June 2021 were selected as the object of study. In the observation group, 25 patients were treated with intensive pain nursing intervention, including 12 males and 13 females; The average age was $(60.12 \pm 3.98)$ years and the body weight was $(58.64 \pm 3.71) \mathrm{kg}$. Control group, with a total of 25 patients, including 14 male, 11 female, used conventional nursing intervention. It's average age was $(60.23 \pm 3.72)$ years, and body weight was $(58.81 \pm 3.64) \mathrm{kg}$. There was no difference in basic data between the two groups $(P>0.05)$, which has comparability. 


\subsection{Methods}

The control group was given routine nursing such as analgesic drugs or analgesic pump, and the observation group was given pain nursing. Patients should be closely monitoring their vital signs after operations, focusing on monitoring blood pressure, heart rate and other indicators. it is necessary to input painkillers to patients, pay attention to the patient's main complaint, and observe the patient's state.

\subsubsection{Education and training of medical staff}

The professional operation of medical staff plays a decisive role in the treatment of patients. The patients in the observation group were treated with standard analgesic pump, and the nurses were trained in pain control. Doctors with anesthesia experience explained the meaning of pain, mastered the classification of pain, defined the steps of pain care and the use of analgesic drugs for the nurses ${ }^{[1]}$. Formulate a phased pain relief plan for patients, inject painkillers into patients according to the nursing plan, strengthen the monitoring of patients' physical indicators, communicate more with patients, ask patients about the effect of painkillers, and conduct the next injection before the pain relief effect of patients is eliminated, so as to maintain the curative effect of drugs in patients and relieve the physical pain and psychological pressure caused by pain. The dose of drugs should be strictly controlled to prevent the body from tolerating drugs, reduce the effect of pain relief, and avoid adverse reactions of patients to drugs ${ }^{[2]}$.

\subsubsection{Popularization of pain}

Introduce the pain level and causes to patients, so that patients can have a certain psychological cognition, introduce the action mode and mechanism of analgesic drugs, and explain the use effect of analgesic drugs. Guide patients to learn some non-drug analgesic relief methods, and explain the possible complications caused by pain to patients. We should evaluate the pain of patients, reasonably control the dosage of drugs according to the state of patients, and give targeted nursing methods to different patients. After effectively alleviating patients' pain, we should timely give patients encouragement and comfort, enhance patients' confidence, and make patients believe that the pain is only temporary, with scientific treatment methods and strict postoperative nursing, the pain will eventually be overcome, the pain will eventually disappear. And make the patients actively cooperate with treatment as well as actively carry out postoperative recovery [3].

\subsubsection{Carry out psychological counseling}

For cancer patients, scientific treatment is only half of the success, while the other half comes from psychological counseling. Regular psychological counseling is applicable to any disease, especially for lung cancer patients. Therefore, it puts forward higher requirements for the professionalism of medical staff [4]. The patient's emotional changes and psychological changes play a certain objective role in the whole course of treatment. When talking, the medical staff should behave naturally, sincerely express their concern and sympathy for the patient, ask for the patient's opinion and provide the needed help, so that the patient has a sense of trust to the nurse, and can tell the nurse about the change in heart. Therefore, capturing patients' anxiety and psychological changes is also one of the daily works of medical staff. Appropriate psychological care is given at the right time to eliminate patients' concerns, stabilize their emotions, stimulate patients' confidence in treatment, and makes patients actively and optimistically cooperate with medical staff. If the patient's anxiety, fear, depression and other negative emotions caused by pain cannot be resolved for a long time, the nursing staff should report in time and take necessary measures for the patient, for example, let the psychologist consult him. Nurses should regularly carry out psychological oneto-one counseling activities for patients. Most lung cancer patients are vulnerable in psychological and physiological aspects. Nurses can take the initiative to communicate with patients, eliminate patients' 
tension, reduce patients' pessimistic expectations and after surgery, guide family members to take good care of patients in life and spirit, and conduct psychological counseling for patients through various means. Play some relaxed music for postoperative patients, relieve their anxiety and tension, and play some wonderful movies, football matches and other films that can attract patients' attention, so as to relieve patients' pain and eliminate adverse factors hindering patients' recovery ${ }^{[5]}$.

\subsubsection{Later recovery}

Cancer patients are different from other patients, their inner activities are more complex, and their later recovery is also more difficult. At the same time, their postoperative recovery also needs strong professional guidance, so medical staff should pay regular return visits to patients to guide their families in some professional postoperative care. Regular return visits by medical staff can greatly reduce the worries of patients during rehabilitation, alleviate the contradictions between patients and their families, and let their families actively participate in helping patients establish anti-cancer beliefs. Every cancer patient's desire for life is very strong. Lung cancer patients are also eager to restore a normal life. What they need is not sympathy, but people's help and support. Therefore, it is necessary to establish a good nurse patient relationship with patients and their families, encourage their families and friends to be considerate and take care of patients, and often visit patients to make patients feel warm ${ }^{[6]}$.

\subsection{Exclusion criteria}

The digital evaluation method was used for pain evaluation. The data of 12 hours, 24 hours and 48 hours after operation were collected for evaluation. The higher the score, the heavier the pain of patients.

\subsection{Statistical analysis}

SPSS24.0 statistical software was used to process the data, and the counting data were expressed in (n $/ \%$ ) and $\chi^{2}$ test, as well as the measurement data are expressed in $\left({ }^{x} \pm \mathrm{s}\right)$ and $\mathrm{t}$ test. As $P<0.05$, the difference is statistically significant.

\section{Results}

Table 1. Comparison of pain improvement effect between the two groups at $12 \mathrm{~h}, 24 \mathrm{~h}$ and $48 \mathrm{~h}$ after surgery $[\bar{x} \pm \mathrm{s} / \mathrm{min}]$

\begin{tabular}{cccc}
\hline Group & $\mathbf{1 2 h}$ & $\mathbf{2 4 h}$ & $\mathbf{4 8 h}$ \\
\hline Observation group $(\mathrm{n}=25)$ & $7.23 \pm 1.52$ & $5.91 \pm 1.06$ & $4.63 \pm 1.35$ \\
Control group $(\mathrm{n}=25)$ & $8.92 \pm 1.60$ & $6.89 \pm 1.64$ & $5.70 \pm 1.59$ \\
t value & 3.829 & 2.509 & 2.565 \\
$P$ values & 0.000 & 0.016 & 0.014 \\
\hline
\end{tabular}

\section{Discussion}

With the development of society, the incidence and mortality of lung cancer are increasing. Especially in developed countries, the incidence rate of lung cancer is even higher. Most lung cancer patients are in the late stage. The existing treatment methods are difficult to control, resulting in higher mortality. According to the unified world cancer mortality rate, lung cancer ranks first ${ }^{[7]}$.

The first choice for clinical treatment of lung cancer is surgical treatment. For early cancer, the survival rate of patients is more than $80 \%$ through effective treatment ${ }^{[8]}$. However, most patients with lung cancer will have complications after surgical treatment. Pain is the main complaint of patients. In addition, patients 
will also have dyspnea and fatigue, especially the elderly and weak may also cause the original diseases such as chronic bronchitis and emphysema, which has a great impact on the postoperative life of patients, and professional nursing intervention is needed. Now, it is relatively simple to evaluate the therapeutic effect of lung cancer from the resection of tumor tissue. The recovery of patients and the rate of complications need to be taken into account. After receiving surgical treatment, lung cancer patients can effectively prolong their survival time. Patients need to be cared for in their postoperative recovery, so that patients can face their postoperative life in a better state in terms of physiology and psychology, so as to improve the effect of surgical recovery. The general routine nursing model has some defects. Pain nursing intervention can effectively alleviate the pain symptoms of patients. In this study, the pain scores of patients in the observation group at 12-48 hours after operation are lower than those in the control group, indicating that intensive pain nursing plays a positive role in postoperative nursing. Secondly, strengthening pain nursing also has a good dredging effect on patients' emotions, which can effectively improve patients' psychological state and improve patients' quality of life. Pain nursing can greatly promote patients' return to normal life after operation. During the implementation of pain care, the nurses are patient-centered for their services. In the service process, the nursing concept of the nurses is more advanced, the nursing intervention path is more standardized, they can have a more professional judgment on the state of the patients, effectively help and guide the patients in terms of pain and psychology. And the patients' satisfaction with clinical nursing work is also improved, which can make the patient enter the normal life state faster.

It can be seen from the above that the effect of postoperative pain nursing for patients is better than that of routine nursing. It can effectively improve the degree of pain and effectively relieve the negative emotions of patients. It has great application value in clinical nursing and is worth popularizing.

\section{Disclosure statement}

The author declares no conflict of interest.

\section{References}

[1] Zhang J, Xu BN, Zhang L, et al. 2019, Application Value of Accelerated Rehabilitation Surgery Combined with Single-port Thoracoscopy in Lung Cancer Surgery. Journal of Modern Oncology, 27(2): 355-358.

[2] Zhang L, 2021, Effect of Psychological Nursing Intervention in Operating Room on Anxiety and Postoperative Pain in Patients with Lung Cancer. Special Health, (14): 283.

[3] Batchelor JPT, Ljungqvist O, 2019, A Surgical Perspective of ERAS Guidelines in Thoracic Surgery. Current Opinion in Anaesthesiology, 32(1): 17-22.

[4] Liu JH, Wang P, Liu Y, 2019, Effects of Psychological Nursing based on Relaxation Training on Pain in Patients undergoing Thoracoscopic Surgery for Non-small Cell Lung Cancer. Hebei Medical Journal, 41(4): 633-636.

[5] Tang SH, 2021, Effect of Rapid Rehabilitation Surgical Nursing on Postoperative Pain and Complications in Patients with Thoracoscopic Lung Cancer Surgery. Health Management, (2): 237.

[6] Liu JH, Wang P, Liu Y, 2019, Effects of Psychological Nursing based on Relaxation Training on Pain in Patients undergoing Thoracoscopic Surgery for Non-small Cell Lung Cancer. Hebei Medical Journal, 41(4): 633-636.

[7] Kulkarni R, et al., 2019, Clinical and Epidemiological Profile of Neuroendocrine Tumors: An Experience from a Regional Cancer Center from Western India. South Asian Journal of Cancer, 8(3):198-202. 
[8] Kong ZQ, Zhi XX, Feng LL, et al., 2021, Application and Efficacy Comparison of Painless Nursing Model in Patients with Lung Cancer Undergoing Thoracoscopic Surgery. Acta Academiae Medicinae Wannan, 40(2): 195-197. 